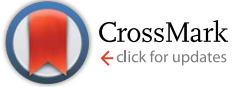

Cite this: RSC Adv., 2017, 7, 4681

Received 10th November 2016 Accepted 2nd January 2017

DOI: $10.1039 / c 6 r a 26587 a$

www.rsc.org/advances

\section{Preparation, characterization and catalytic performance of HPW/aEVM catalyst on oxidative desulfurization}

\author{
Pengcheng Huang, ${ }^{a}$ Guangqin Luo, ${ }^{a}$ Lihua Kang, ${ }^{\text {ab }}$ Mingyuan Zhu*ab and Bin Dai ${ }^{\text {ab }}$ \\ Expanded vermiculite (EVM) was prepared, treated with acid (aEVM) and incorporated with $\mathrm{H}_{3} \mathrm{PW}_{12} \mathrm{O}_{40}$ \\ (HPW) by an ordinary impregnation method. Various characterizations of the HPW/aEVM catalyst \\ indicated that the HPW was dispersed homogeneously on the aEVM support. The catalytic activity of the \\ HPW/aEVM catalyst was evaluated in the catalytic oxidation process of dibenzothiophene (DBT), and the \\ optimal reaction conditions were investigated, including the loading quantity of the phosphotungstic \\ acid, the reaction temperature and time, the $\mathrm{O} / \mathrm{S}\left(\mathrm{H}_{2} \mathrm{O}_{2} /\right.$ sulfur $)$ molar ratio, and the catalyst dosage. \\ Under the experimental conditions, the sulfur removal rate for model oil was almost $100 \%$. After 7 \\ recycles, the loss of catalytic activity was only 99.95 to $98.06 \%$.
}

\section{Introduction}

The existence of sulfur compounds in fuel oil is the main source of $\mathrm{SO}_{x}$ emissions. The emissions of $\mathrm{SO}_{x}$ not only cause acid rain but also reduce the conversion efficiency of automobile exhaust converters for pollutants. Because of increasing environmental problems in recent years, most countries enforce strict regulations for the sulfur content in fuel oil. Oxidative desulfurization (ODS) processes have advantages such as mild reaction conditions, simple operation processes, good desulfurization effects, and have become one of the most promising deep desulfurization methods in recent years. ${ }^{1}$

In this study, the ODS process was investigated with oxidants, extraction agents and various catalysts, including ionic liquids, ${ }^{2}$ organic acids, ${ }^{3}$ inorganic acids ${ }^{4}$ and heteropoly acids (HPAs). ${ }^{5}$ Among these catalysts, the Keggin structure possessed by some HPA catalysts were proven to be very effective for the ODS of fuel oil. ${ }^{6}$

However, the heteropoly acid catalyst easily dissolves and is difficult to separate and recover for the next use. For this reason, HPAs should be immobilized on a solid support to provide the stable and water-tolerant ability of the catalyst. Many researchers tried to solve this problem by immobilizing HPW on the surface of a solid support, such as $\mathrm{SiO}_{2},{ }^{7}$ TUD-1. ${ }^{8}$ and SBA15. ${ }^{9}$ With simple filtration, the solid catalyst can be reused for the cycles of the ODS process. In our previous study, we observed that amino-functionalized MCM-41 and mesoporous

${ }^{a}$ School of Chemistry and Chemical Engineering, Shihezi University, Shihezi, Xinjiang 832003, P. R. China. E-mail: zhuminyuan@shzu.edu.cn; Fax: +86 9932057210; Tel: $+869932057270$

${ }^{b}$ Key Laboratory for Green Processing of Chemical Engineering of Xinjiang Bingtuan, Shihezi, Xinjiang 832003, P. R. China graphitic carbon act as the support of HPAs, and an excellent recycle rate can be obtained for the ODS process of fuel oil. However, The MCM-41 molecular sieve and mesoporous graphitic carbon are not cost effective because of their complicated synthesis process.

Vermiculite (VMT) is a natural mineral that is inexpensive and abundant in China, especially in the Xinjiang province. VMT has typical features, such as a lamellar structure and high ion exchange capacity, which allows interactions with organic substances. ${ }^{10}$ Actually, VMT has been well investigated in different catalysis areas, such as DeNOx processes, ${ }^{11}$ photocatalysis $^{12}$ and as catalysts for ethanol oxidation. ${ }^{13}$ Therefore, it was inferred that VMT may serve as the support of HPAs and applied in ODS processes. In this study, we synthesized a HPW/ aEVM catalyst, characterized it and evaluated its catalytic performance. The aim of this work was to develop a new catalyst that is cost effective and has potential applications in the oxidative desulfurization of fuel oil.

\section{Experimental section}

\subsection{Pretreatment of VMT}

Raw VMT samples were supplied by Xinjiang YuliXinlong Vermiculite Co., Ltd., China, The element composition of raw VMT and aEMV are given in Table 1. The preparation method for HPW/aEVM catalysts was very similar to that as reported in the literature. ${ }^{14,15}$ Raw VMT $(150 \mathrm{~g})$ were first treated with hydrogen peroxide $(30 \%$ concentration, $750 \mathrm{ml})$ at $353 \mathrm{~K}$ for $2 \mathrm{~h}$ and then dried at $800 \mathrm{~W}$ in a microwave oven for $1 \mathrm{~min}$. Then, the dried EVM was ground in a pulverizer and sieved through a 120-mesh sieve. The acid treatment processes were conducted by placing the obtained EVM ( $1 \mathrm{~g})$ powder in a chloroazotic acid solution $(20 \mathrm{ml})$ with different 
concentrations in the ranges of 0.5 to $6 \mathrm{~mol} \mathrm{~L}^{-1}$. The mixture was placed under mechanical stirring at $353 \mathrm{~K}$ for $5 \mathrm{~h}$, and after the mixture cooled to room temperature, solid precipitates were collected through a centrifugal method and washed several times with deionized water until they were chloridefree. ${ }^{16}$ The samples were dried overnight at $373 \mathrm{~K}$ to yield the aEVM.

\subsection{HPW/aEVM catalyst preparation}

$\mathrm{H}_{3} \mathrm{PW}_{12} \mathrm{O}_{40}(0.25 \mathrm{~g})$ was first dissolved in purified water $(10 \mathrm{ml})$ and then placed the aEVM powder $(1 \mathrm{~g})$ to the phosphotungstic acid solution with vigorous stirring for $24 \mathrm{~h}$ under $333 \mathrm{~K}$. The solid samples were collected by filtration, washed with purified water and then dried at $383 \mathrm{~K}$ to yield the $20 \% \mathrm{HPW} / \mathrm{aEVM}$ catalyst. The $10 \%$ and $30 \%$ HPW/aEVM catalysts were obtained only by varying the amounts of phosphotungstic acid.

\subsection{Characterization}

Brunauer-Emmett-Teller (BET) surface area analysis was conducted by using a Micromeritics Model ASAP 2020 instrument at $77 \mathrm{~K}$ to obtain the nitrogen adsorption isotherms. All VMT samples were degassed for $6 \mathrm{~h}$ under a vacuum at $383 \mathrm{~K}$. FT-IR spectra were conducted by a Nicolet AVATAR 360. The transmission electron microscopy (TEM) analysis results were obtained by using a Tecnai F30 field emission TEM. Inductively coupled plasma atomic emission spectroscopy (ICP-AES) experiments were conducted on a thermo scientific ICAP 6000 series ICP spectrometer. The XRD pattern data were obtained on a Bruker advanced D8 X-ray diffractometer equipped with a Cu-K $\alpha$ irradiation $(\lambda=1.5406 \AA)$ as source at $40 \mathrm{~mA}$ and $40 \mathrm{kV}$.

\subsection{Catalytic ODS process}

The oxidative desulfurization process was conducted at $333 \mathrm{~K}$ in a three-necked flask accompanied by a condensing system. Model oils with $100 \mathrm{ppm}$ sulfur concentration were obtained by dissolving dibenzothiophene (DBT), 4,6-dimethyldibenzothiophene (4,6-DMDBT), thiophene (Th), and benzothiophene (BT) compounds into $10 \mathrm{ml} n$-octane in a $100 \mathrm{ml}$ three-necked flask. The ODS system included model oils, oxidant $\mathrm{H}_{2} \mathrm{O}_{2}$, and the HPW/aEVM catalyst. All components were mixed in a threenecked flask and fixed in a water bath under magnetic stirring and reflux. After reaching the optimal reaction time, the upper clarified model oil was collected and extracted by methanol. Then, the sulfur content was detected by a microcoulometric detector (WK-2D). The solid catalyst was recycled by suction filtration and washed with methanol.

\section{Results and discussion}

\subsection{The concentration of acid treatment options}

The specific surface area analysis results of the EVM treated with different acid concentrations are presented in Table 2. Based on the data, the surface area of the EVM was only $4.42 \mathrm{~m}^{2}$ $\mathrm{g}^{-1}$, however, after treated with an acid solution of $1.5 \mathrm{~mol} \mathrm{~L}^{-1}$, the surface area increased to $714.81 \mathrm{~m}^{2} \mathrm{~g}^{-1}$. The $S_{\mathrm{BET}}$ result of the leached samples increased with the acid concentrations changed from 0.5 to $2 \mathrm{~mol} \mathrm{~L}^{-1}$. However, when the acid concentrations increased from 2 to $6 \mathrm{~mol} \mathrm{~L}^{-1}$, the $S_{\mathrm{BET}}$ result gradually decreased, which illustrated that the micropores formed by the leaching of the octahedral layers were unstable and decreased rapidly at high acid concentrations. ${ }^{17}$

Moreover, as the acid concentration increased, the removal rate of the sulfocompound increased gradually. When the acid solution reached $1.5 \mathrm{~mol} \mathrm{~L}^{-1}$, the sulfur removal rate was almost $99.89 \%$. Since the desulfurization rate did not vary significantly with the improvement of acid concentration over $1.5 \mathrm{~mol} \mathrm{~L}^{-1}$, we choose the concentration of $1.5 \mathrm{~mol} \mathrm{~L}^{-1}$ as an optimal experimental condition.

\subsection{Characterization of the catalyst}

Fig. 1 shows the X-ray diffraction patterns of the HPW, raw VMT, EVM and HPW/aEVM samples with different contents of HPW (10-30\%). The XRD patterns of the raw material (Fig. 1B), shows the characteristic peaks of vermiculite. ${ }^{18}$ In Fig. 1A we observed a broad peak at $2 \theta=23.3^{\circ}$ in the aEVM samples, which proved that the mesoporous silica of the aEVM was amorphous. ${ }^{\mathbf{1 9}}$ These characteristic peaks were also observed in the other three HPW/ aEVM catalysts. However, different contents of the phosphotungstic acid catalyst showed no characteristic diffraction peaks of HPW, which proved that the HPW was dispersed homogeneously on the aEVM support.

The FT-IR spectra of EVM, HPW and HPW/aEVM samples with different contents of HPW are shown in Fig. 2. We

Table 2 Surface area of vermiculite and conversion of DBT treated with different acid concentration

\begin{tabular}{lrl}
\hline $\begin{array}{l}\text { Acid concentration } \\
\left(\mathrm{mol} \mathrm{L}^{-1}\right)\end{array}$ & $\begin{array}{l}S_{\mathrm{BET}} \\
\left(\mathrm{m}^{2} \mathrm{~g}^{-1}\right)\end{array}$ & $\begin{array}{l}\text { Conversion of DBT } \\
(\%)\end{array}$ \\
\hline 0 & 4.42 & 63.11 \\
0.5 & 6.50 & 65.20 \\
1 & 325.57 & 76.23 \\
1.5 & 714.81 & 99.89 \\
2 & 720.12 & 100 \\
3 & 628.24 & 100 \\
6 & 622.30 & 99.99
\end{tabular}

Table 1 Element composition of the raw vermiculite and 20\% HPW/aEVM

\begin{tabular}{|c|c|c|c|c|c|c|c|c|c|c|}
\hline Sample & $\mathrm{O}$ & $\mathrm{Si}$ & $\mathrm{Mg}$ & $\mathrm{Al}$ & K & $\mathrm{Fe}$ & $\mathrm{Na}$ & $\mathrm{Ti}$ & $\mathrm{Ca}$ & $\mathrm{W}$ \\
\hline Raw VMT (wt\%) & 39.21 & 23.13 & 17.69 & 8.35 & 5.06 & 3.53 & 1.24 & 1.07 & 0.72 & - \\
\hline 20\% HPW/aEVM (wt\%) & 45.39 & 49.75 & 1.14 & 1.07 & 0.34 & 0.68 & 0.05 & 0.43 & 0.13 & 1.02 \\
\hline
\end{tabular}



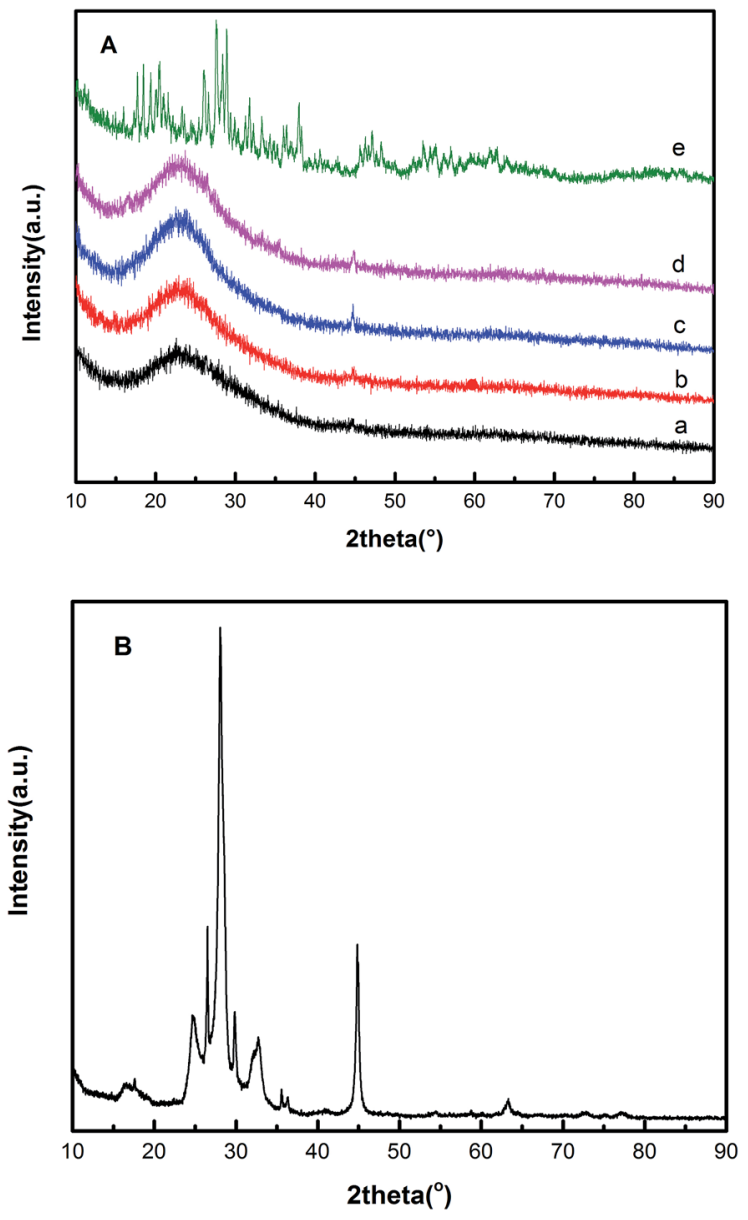

Fig. 1 (A) The wide-angle XRD patterns of (a) aEVM, (b) 10\% HPW/ aEVM, (c) $20 \% \mathrm{HPW} / \mathrm{aEVM}$, (d) 30\% HPW/aEVM and (e) HPW. (B) XRD patterns of raw VMT.

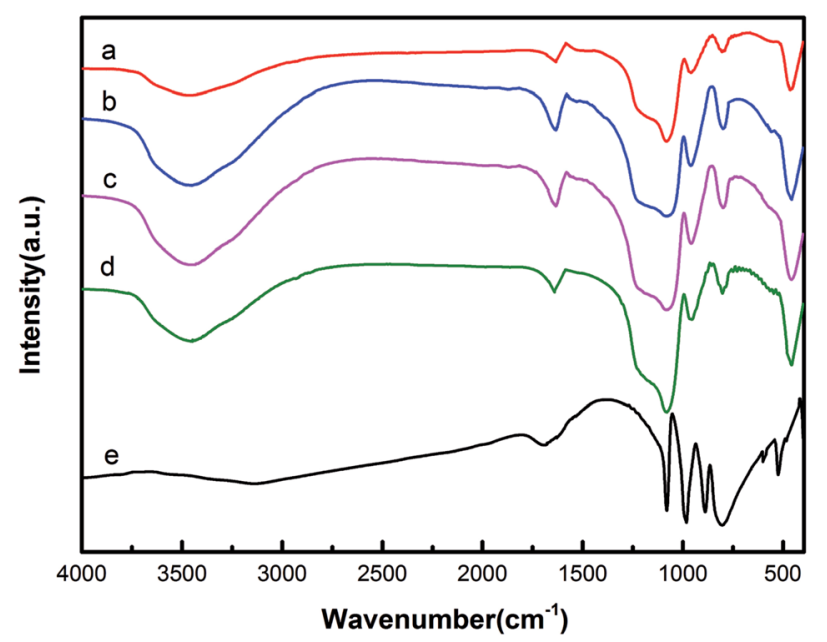

Fig. 2 FT-IR spectra of (a) aEVM, (b) 10\% HPW/aEVM, (c) 20\% HPW/ aEVM, (d) 30\% HPW/aEVM and (e) HPW.

observed that the pure HPW samples with Keggin structures presented four strong IR peaks at $802 \mathrm{~cm}^{-1}$ and $889 \mathrm{~cm}^{-1}$ (corresponding to the $\mathrm{W}-\mathrm{O}_{\mathrm{c}}-\mathrm{W}$ and $\mathrm{W}-\mathrm{O}_{\mathrm{b}}-\mathrm{W}$ bands,
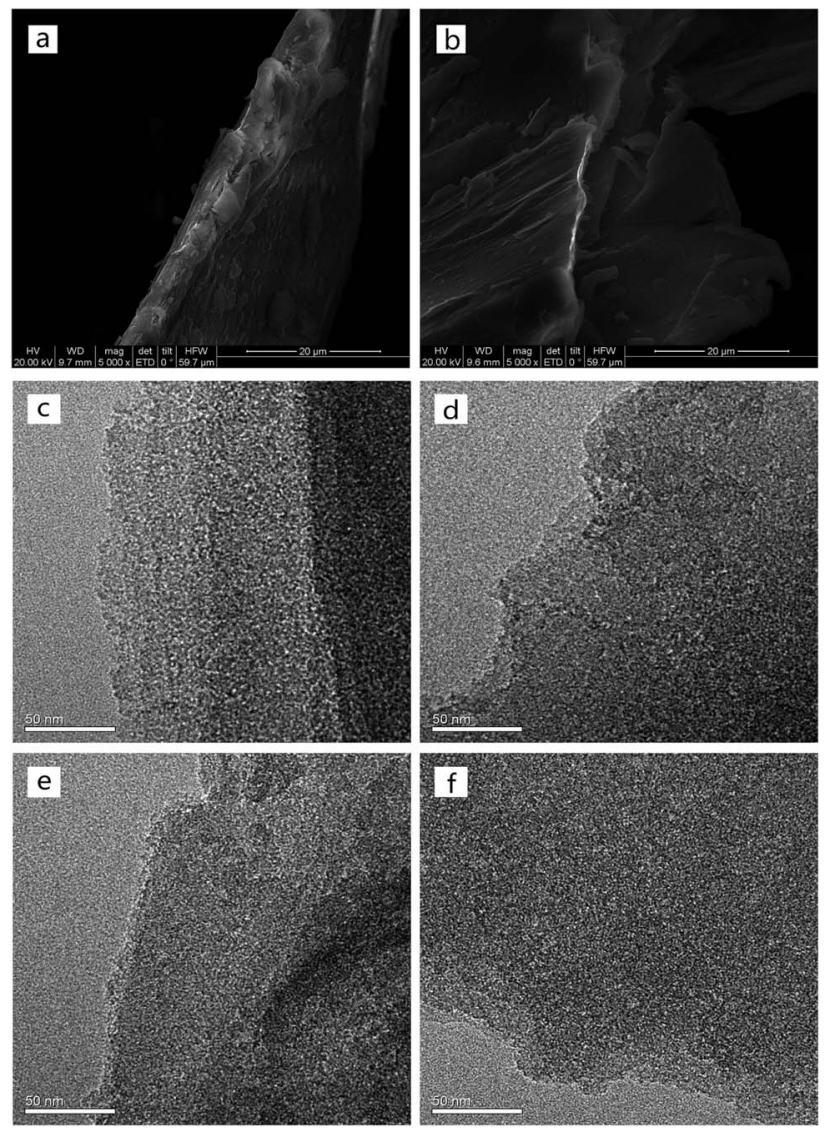

Fig. 3 SEM images of (a) raw VMT, (b) EVM, and TEM images of (c) aEVM, (d) 10\% HPW/aEVM, (e) 20\% HPW/aEVM, (f) 30\% HPW/aEVM.

respectively), $981 \mathrm{~cm}^{-1}$ (terminal bands for $\mathrm{W}=\mathrm{O}$ in the exterior $\mathrm{WO}_{6}$ octahedron), and $1080 \mathrm{~cm}^{-1}$ (stretching frequency of $\mathrm{P}-\mathrm{O}$ in the central $\mathrm{PO}_{4}$ tetrahedron). ${ }^{20}$ The aEVM sample displayed the band (at 460,802 , and $1078 \mathrm{~cm}^{-1}$ ) that corresponded to the vibrational modes of $\mathrm{SiO}_{2}$. The bands at 802 and 1078 $\mathrm{cm}^{-1}$ corresponded to the symmetric and anti-symmetric stretching frequency of $\mathrm{Si}-\mathrm{O}-\mathrm{Si}$. The bands at $952 \mathrm{~cm}^{-1}$ corresponded to the stretching frequency of Si-O-H. ${ }^{21}$ For the HPW/ aEVM sample, no significant differences of characteristic bands were observed after loading the phosphotungstic acid. In particular, the $\mathrm{P}-\mathrm{O}$ band of HPW was not clearly observed in the HPW/aEVM samples because of the coverage of the broad Si-OSi band. ${ }^{22}$ In addition, the bands of the 20\% HPW/aEVM samples were slightly strengthened compared with those of the unsupported samples. This further proved the incorporation of HPW into aEVM frameworks.

Fig. 3 shows the morphologies of raw VMT, EVM and HPW/ aEVM samples with different contents of HPW. As shown in Fig. 3a and b, EVM was successfully prepared by placing the raw VMT into the $\mathrm{H}_{2} \mathrm{O}_{2}$ aqueous solution bath. The expanded samples exhibited obvious interlayer gallery structures. After acid treatment, channels formed on the surface of the EVM sample, but the layered framework was retained (Fig. 3c). When loading on the phosphotungstic acid, the channel structure remained but the HPW species were not observed (Fig. 3d-f), 

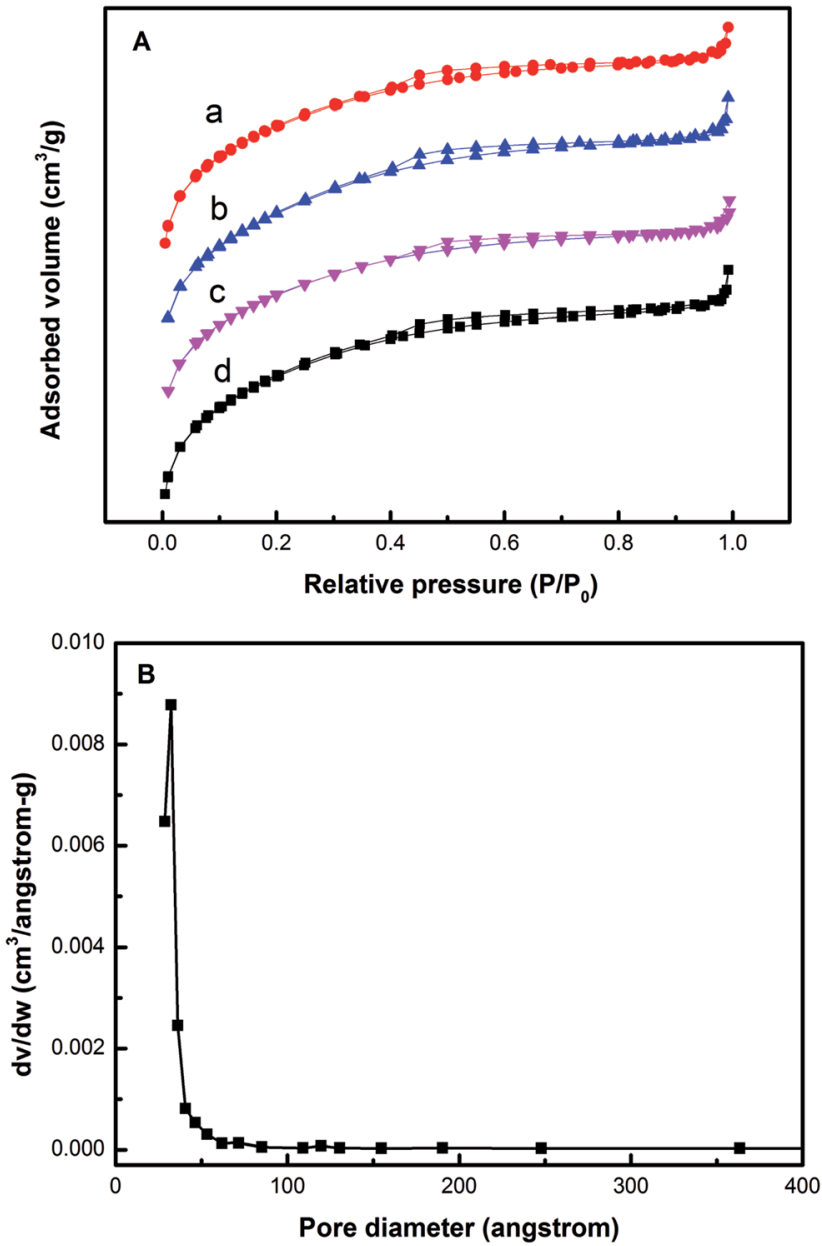

Fig. 4 (A) $\mathrm{N}_{2}$ adsorption-desorption isotherms of (a) 10\% HPW/aEVM, (b) 20\% HPW/aEVM, (c) 30\% HPW/aEVM and (d) aEVM. (B) Pore size distribution curve of $20 \% \mathrm{HPW} / \mathrm{aEVM}$.

which suggested that the phosphotungstic acids were dispersed homogeneously on the channel and layer of aEVM.

The $\mathrm{N}_{2}$ adsorption/desorption isotherms of the aEVM and HPW/aEVM samples with different contents of HPW are shown in Fig. 4A. The four samples presented an hysteresis loop range from 0.4 to 0.8 relative pressure, and the isotherm patterns were similar to type IV isotherm. These characteristics corresponded to the mesoporous materials with the pore channel formed in the interlayer framework. ${ }^{20}$ Fig. 4B shows the pore size distribution curve of $20 \% \mathrm{HPW} / \mathrm{aEVM}$, the average pore size is very uniform and centered at around $2.39 \mathrm{~nm}$. The physical-chemical parameters of the four aEVM samples are shown in Table 3.

Table 3 Physico-chemical parameters of various samples

\begin{tabular}{llll}
\hline Sample & $S_{\text {BET }}\left(\mathrm{m}^{2} \mathrm{~g}^{-1}\right)$ & $V_{\mathrm{P}}\left(\mathrm{cm}^{3} \mathrm{~g}^{-1}\right)$ & $D(\mathrm{~nm})$ \\
\hline aEVM & 714.81 & 0.413 & 2.44 \\
$10 \%$ HPW/aEVM & 682.65 & 0.409 & 2.39 \\
$20 \%$ HPW/aEVM & 676.31 & 0.399 & 2.39 \\
$30 \%$ HPW/aEVM & 624.01 & 0.373 & 2.23
\end{tabular}

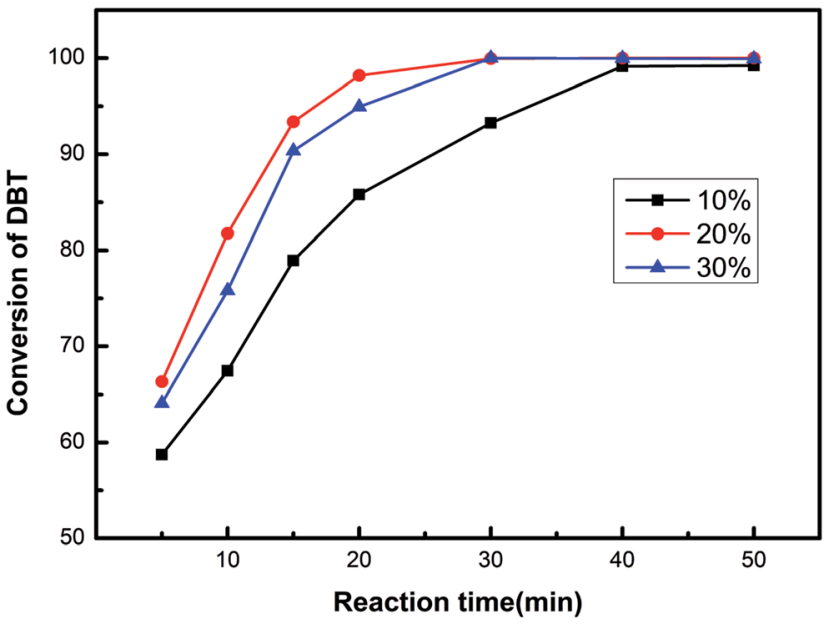

Fig. 5 The conversion of DBT of the HPW/aEVM catalysts with different loading. $T=333 \mathrm{~K}, \mathrm{O} / \mathrm{S}=6$, catalyst dosage $=0.04 \mathrm{~g} / 10 \mathrm{ml}, t$ $=0.5 \mathrm{~h}$.

Compared with those of the aEVM samples, the $S_{\mathrm{BET}}$ and $V_{\mathrm{P}}$ results of the HPW/aEVM samples decreased with the content increase of HPW, which corresponded to the blockage of heteropolyanions on the mesopores structure of the support.

\subsection{Catalytic results}

The catalytic effects of the HPW/aEVM catalyst with different HPW loadings were evaluated on a three-necked flask by dissolving DBT in normal octane as the model fuel oil. As shown in Fig. 5, the DBT conversion follows the order of $20 \% \mathrm{HPW} / \mathrm{aEVM}$ $>30 \% \mathrm{HPW} / \mathrm{aEVM}>10 \% \mathrm{HPW} / \mathrm{aEVM}$. These results demonstrated that different loading contents of active tungsten species may have influenced the experimental results.

When the loading content increased from $10 \%$ to $20 \%$, the catalytic effect was obviously enhanced. A complete conversion of DBT $(100 \%)$ was obtained in $0.5 \mathrm{~h}$, with the HPW content reached $20 \%$. This phenomenon was attributed to the high dispersion of HPW on the aEVM support. However, when the HPW contents increased to $30 \%$, the desulfurization effect slightly decreased, which was attributed to the blockage of HPW on the pore channel. These performances were conform to the BET analysis results, wherein the $S_{\mathrm{BET}}$ and $V_{\mathrm{P}}$ reduced as the HPW contents increased. Since the mesoporous pores provided the transferring channel for the sulfocompound, high contents of HPW may block the mesoporous pores, which was the primary cause of the decrease in catalytic activity. Consequently, the $20 \%$ HPW/aEVM catalyst was chosen for the subsequent ODS process.

Fig. 6 shows the four main reaction conditions. Fig. 6A shows the conversion of DBT oxidated to its corresponding sulfones increased with the increment of reaction times. The conversion rate was almost $100 \%$ with $0.5 \mathrm{~h}$. The optimal dosage of the HPW/aEVM catalyst in the ODS process with an organic sulfur concentration of $100 \mathrm{ppm}$ was presented in Fig. 6B. The desulfurization conversion increased with the increment of the catalyst dosage. When the dosage of catalyst increased to $40 \mathrm{mg} /$ 

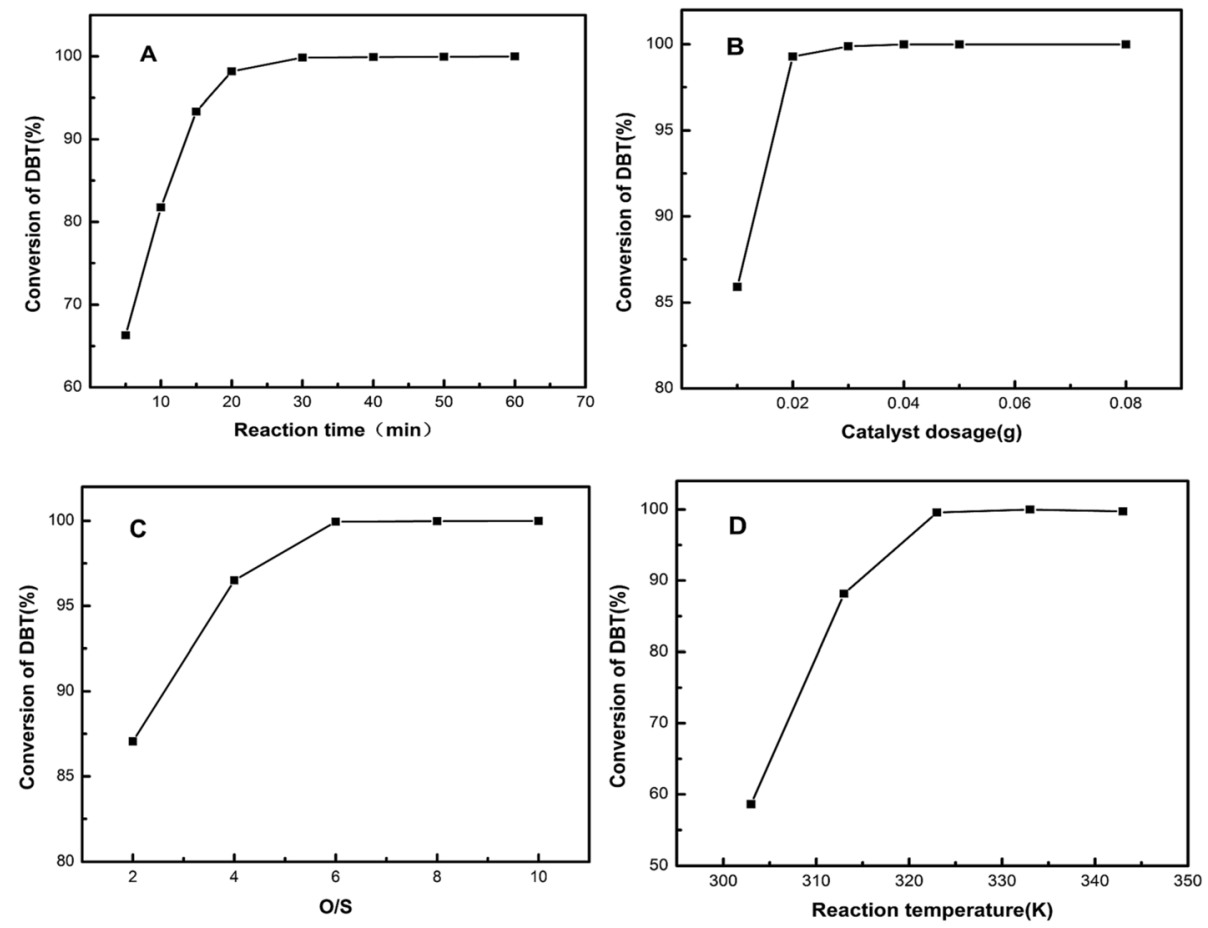

Fig. 6 Effect of the (A) reaction time, (B) catalyst dosage, (C) O/S molar ratio and (D) reaction temperature on the conversion of DBT. $T=333 \mathrm{~K}$, $\mathrm{O} / \mathrm{S}=6$, catalyst dosage $=0.04 \mathrm{~g} / 10 \mathrm{ml}, t=0.5 \mathrm{~h}$.

$10 \mathrm{ml}$, the curve was close to saturation. This result demonstrated that sufficient number of active sites could be provided with the catalyst concentration of $40 \mathrm{mg} / 10 \mathrm{ml}$. Fig. 6C shows the catalytic activities of the $20 \% \mathrm{HPW} / \mathrm{aEVM}$ catalyst with the $\mathrm{O} / \mathrm{S}$ mole ratio range from 2 to 10 . The conversion of DBT significantly increased with the improvement of $\mathrm{O} / \mathrm{S}$ molar ratio. When the ratio reached 6 , the desulfurization conversion was almost $100 \%$ and remained constant above this ratio. However, the value for oxidize DBT to its corresponding sulfone was much higher than the stoichiometric value $\mathrm{O} / \mathrm{S}=2$, because the excess hydrogen peroxide supplemented the consumption of thermal decomposition. ${ }^{23}$ The effect of temperature on the ODS process was investigated as shown in Fig. 6D. In the range of $303 \mathrm{~K}$ to $343 \mathrm{~K}$, the reaction was performed with $\mathrm{O} / \mathrm{S}=6$. The conversion of DBT increased from $58.62 \%$ to $99.98 \%$ when the temperature increased from $303 \mathrm{~K}$ to $333 \mathrm{~K}$. This process was attributed to the peroxo metal complex $\mathrm{W}\left(\mathrm{O}_{2}\right)_{n}$ in HPW active species increased with improving the reaction temperature. ${ }^{22}$ The ODS process depended on $\mathrm{W}\left(\mathrm{O}_{2}\right)_{n}$ as the primary active component for the oxidation of sulfide. When increasing the temperature to $343 \mathrm{~K}$, the desulfuration effect had a slight decrease. This was owing to $\mathrm{H}_{2} \mathrm{O}_{2}$ decomposing and the decrease of the $\mathrm{W}\left(\mathrm{O}_{2}\right)_{n}$ complex with a higher temperature.

Kinetic experiments were performed to better illustrate the reaction activation energy. The ODS process followed pseudofirst-order reaction kinetics, with eqn (1).

$$
r=-\frac{\mathrm{d} c_{t}}{\mathrm{~d} t}=k c_{t}
$$

According to Fig. 7A, the reaction rate constant $(k)$ was obtained by the slope of the linear plot of $\ln \left(c_{0} / c_{t}\right)$ versus reaction time $t$ from eqn (2).
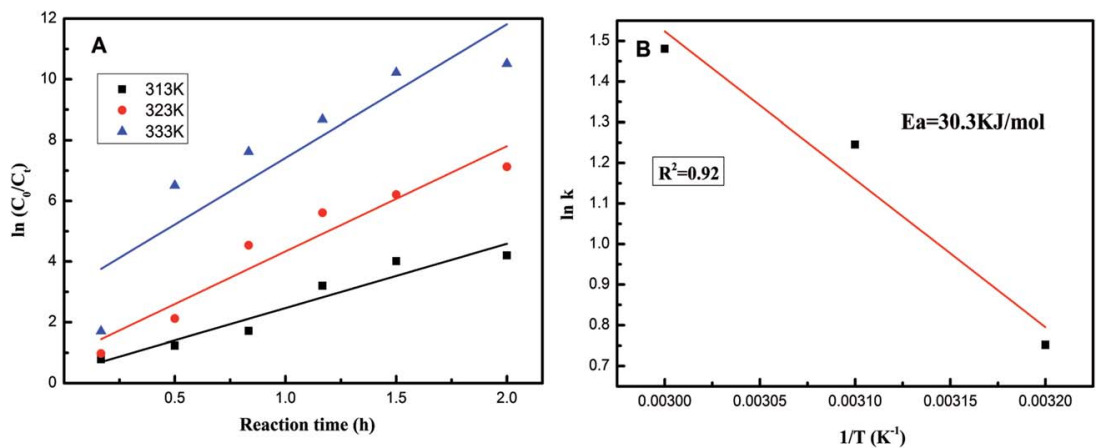

Fig. 7 (A) Fitting of experimental data to the pseudo-first-order rate model. (B) Arrhenius activation energies for DBT of 20\% HPW/aEVM catalyst. 


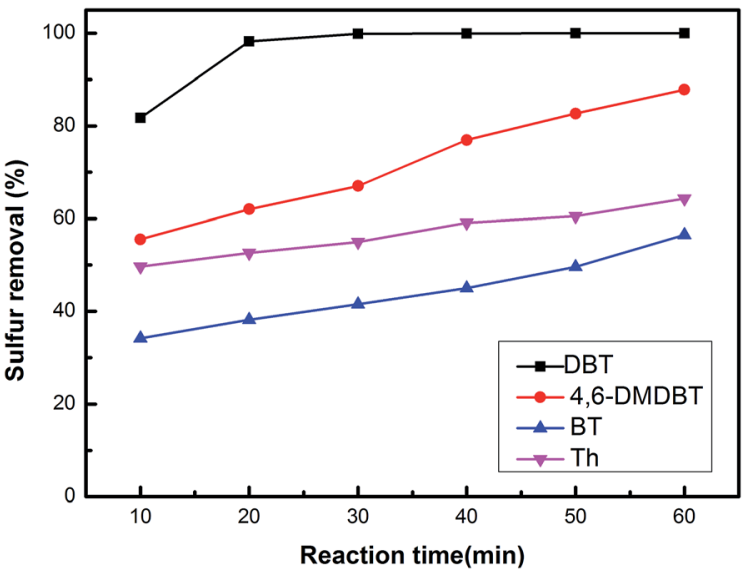

Fig. 8 The sulfur removal of different substrate with 20\% HPW/aEVM as catalyst: $T=333 \mathrm{~K}, \mathrm{O} / \mathrm{S}=6$, catalyst dosage $=0.04 \mathrm{~g} / 10 \mathrm{ml}, t=$ $0.5 \mathrm{~h}$.

$$
\ln \left(c_{t} / c_{0}\right)=-k t
$$

The apparent activation energy for the ODS process was obtained according to various $(k)$ values obtained at different temperatures with eqn (3).

$$
\ln k=\ln A-\frac{E_{\mathrm{a}}}{R T}
$$

As shown in Fig. 7B, according to the Arrhenius equation, we calculated the apparent activation energy for the oxidative desulfurization of DBT to be $30.3 \mathrm{~kJ} \mathrm{~mol}^{-1}$. This result was slightly lower than the previously reported value, ${ }^{24}$ which indicated that the HPW/aEVM catalyst had excellent catalytic activity.

Fig. 8 shows the catalytic oxidation results of different sulfur substrates in the model oil. The oxidative desulfurization activity followed the order DBT $>4,6$-DMDBT $>$ Th $>$ BT. This

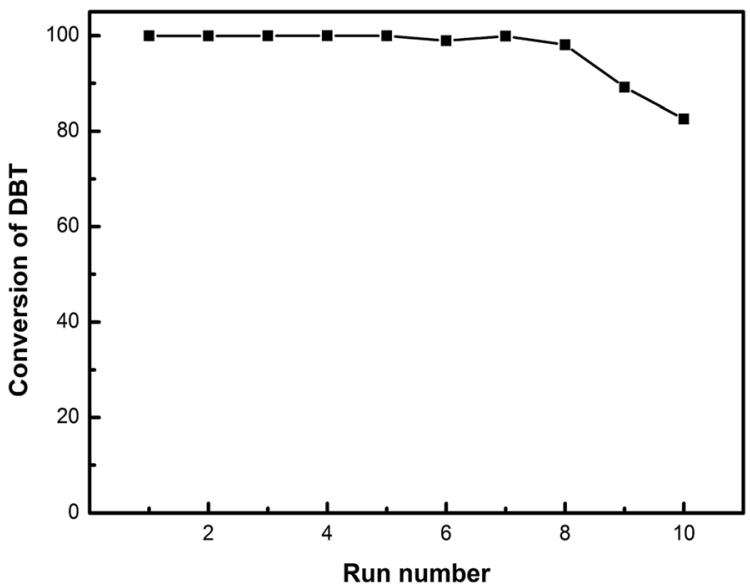

Fig. 9 Effect of the recycles on the conversion of DBT with $20 \%$ HPW/ aEVM as catalyst. was attributed to different sulfides that varied in electron densities of the sulfur atom. The conversion of DBT reached nearly $100 \%$ within $0.5 \mathrm{~h}$, while BT reached only $41.54 \%$, which was consistent with the electron densities of DBT (5.758) being higher than BT (5.739). Similar electron densities (5.760 versus 5.758) existed for 4,6-DMDBT and DBT on the sulfur atom but the oxidative desulfurization activity exhibited a significant difference. The conversion of 4,6-DMDBT reached only $67.01 \%$ within $0.5 \mathrm{~h}$, it might be the methyl groups that caused steric hindrance. The methyl groups of 4,6-DMDBT become an obstacle for the catalytic active site of the catalyst to approach the sulfur atom.

The 20\% HPW/aEVM catalyst was recycled to investigate the reusability and stability, and with each recycle, the catalyst was filtered, washed with methanol, and dried at 353 K. Fig. 9 shows the desulphurization effect of the fresh and spent catalyst after 1-7 recycles. The conversion of DBT decreased from $99.95 \%$ to 98.06\% after 7 recycles, which indicated that the 20\% HPW/ aEVM catalyst had excellent regenerability and the HPW species acted as the active component for the sulfur compounds oxidated to its corresponding sulfones.

To investigate the $\mathrm{W}$ and $\mathrm{P}$ contents and calculate the corresponding content of HPW in the 20\% HPW/aEVM catalyst after 1-7 recycles, we performed an ICP-AES experiment. As shown in Table 4 , the atomic ratio of $\mathrm{P}: \mathrm{W}(1: 11.5)$ was close to the stoichiometric ratio $(1: 12)$. Moreover, the $\mathrm{W}$ and $\mathrm{P}$ content in the fresh $20 \% \mathrm{HPW} / \mathrm{aEVM}$ catalyst were $11.74 \%$ and $0.17 \%$, respectively. The catalytic activity was not significantly reduced after 7 reaction cycles, because the phosphotungstic acid radical ion incorporated into the interlayer region by electrostatic attraction and was constrained by the layer structure. Moreover, for the recycling of $20 \% \mathrm{HPW} / \mathrm{aEVM}$ catalyst, the leaching of tungsten species was only $1.94 \%$ after 7 reaction cycles, which demonstrated that the HPW was incorporated in the channel formed in the surface and interlayer of the aEVM.

The catalytic activity decreased with the leaching of active component. To investigate the leaching effect, we conducted

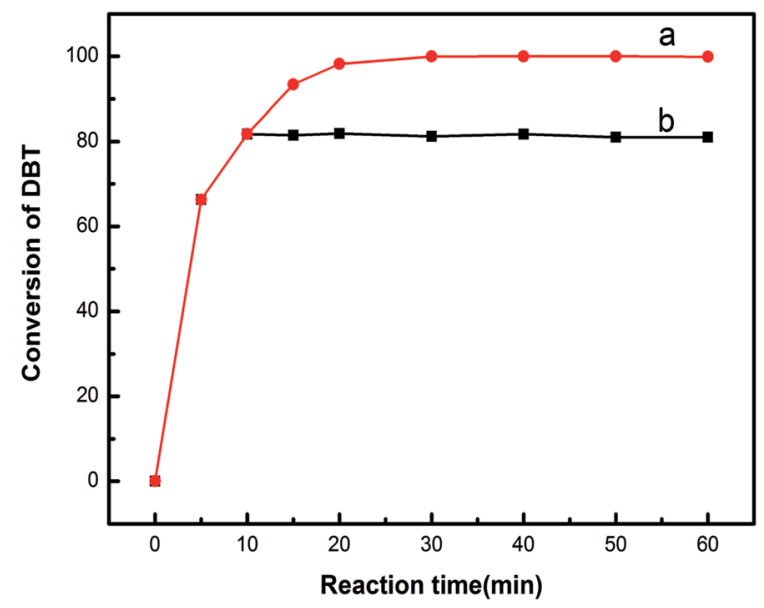

Fig. 10 Hot filtration experiment of 20\% HPW/aEVM catalyst. (a) 20\% $\mathrm{HPW} / \mathrm{aEVM}$ was hot filtrated at 10 minute, (b) with the presence $20 \%$ $\mathrm{HPW} / \mathrm{aEVM}$ as catalyst. 
Table 4 Reusability of $20 \%$ HPW/aEVM ${ }^{a}$

\begin{tabular}{lllll}
\hline $\begin{array}{l}\text { Run } \\
\text { number }\end{array}$ & $\begin{array}{l}\mathrm{P} \\
(\mathrm{wt} \%)^{b}\end{array}$ & $\begin{array}{l}\mathrm{W} \\
(\mathrm{wt} \%)^{b}\end{array}$ & $\begin{array}{l}\text { Molar ratio } \\
\text { of } \mathrm{P}: \mathrm{W}^{c}\end{array}$ & $\begin{array}{l}\text { HPW } \\
(\mathrm{wt} \%)^{c}\end{array}$ \\
\hline $\begin{array}{l}\text { Fresh } \\
\text { catalyst }\end{array}$ & 0.172 & 11.74 & $1: 11.5$ & 15.33 \\
1 cycle & 0.164 & 11.38 & $1: 11.7$ & 14.86 \\
2 cycle & 0.153 & 11.23 & $1: 11.8$ & 13.97 \\
3 cycle & 0.150 & 10.55 & $1: 11.6$ & 13.78 \\
4 cycle & 0.143 & 10.09 & $1: 11.9$ & 13.17 \\
5 cycle & 0.140 & 9.76 & $1: 12.1$ & 12.75 \\
6 cycle & 0.134 & 9.35 & $1: 11.9$ & 12.21 \\
7 cycle & 0.129 & 8.54 & $1: 11.1$ & 11.15 \\
8 cycle & 0.116 & 7.87 & $1: 11.4$ & 10.27 \\
9 cycle & 0.112 & 7.52 & $1: 11.3$ & 9.82
\end{tabular}

${ }^{a}$ Reaction conditions: reaction temperature $333 \mathrm{~K}$, reaction time $2.5 \mathrm{~h}$, $\mathrm{O} / \mathrm{S}=6$, catalyst dosage $=0.04 \mathrm{~g} / 10 \mathrm{ml} .{ }^{b}$ As determined by ICP-AES experiments. ${ }^{c}$ As calculated from the ICP-AES results, the atomic weight of $\mathrm{P}$ and $\mathrm{W}$, and molecular weight of HPW.

a hot filtration experiment. When the reaction lasted for $10 \mathrm{~min}$, the catalyst was filtered out to compare the desulphurization effect with the presence of the 20\% HPW/aEVM catalyst. As shown in Fig. 10, the desulfurization rate slightly increased from $81.75 \%$ to $81.89 \%$ with reaction times from 10 to $60 \mathrm{~min}$. However, the desulfurization rate was much lower than the existence of the $20 \% \mathrm{HPW} / \mathrm{aEVM}$ catalyst, which showed that the leaching content of the active component from the silica frameworks of the aEVM material was small in scope and consistent with the ICP-AES test.

\section{Conclusions}

In conclusion, an inexpensive and highly efficient HPW/aEVM catalyst was successfully prepared and applied to the oxidation desulfurization process. Under the optimized conditions, the sulfur content efficiently decreased from 100 to $0.047 \mathrm{ppm}$ within $0.5 \mathrm{~h}$, which corresponded to nearly $100 \%$ sulfur removal rate. After 7 recycles, the catalytic activity of the 20\% HPW/ aEVM catalyst decreased by $1.89 \%$, which indicated an inexpensive and promising catalyst for the deep desulfurization of model fuel.

\section{Acknowledgements}

This work was supported by the Innovation Funds for Distinguished Young Scientists of Xinjiang Bingtuan (2011CD001), and the Foundation of Young Scientist in Shihezi University (No. 2013ZRKXJQ03).

\section{References}

1 M. A. Rezvani, A. F. Shojaie and M. H. Loghmani, Catal. Commun., 2012, 25, 36-40.

2 H. Gao, C. Guo, J. Xing, J. Zhao and H. Liu, Green Chem., 2010, 12, 1220-1224.

3 D. Zhao, J. Wang and E. Zhou, Green Chem., 2007, 9, 12191222.

4 P. S. Tam, J. R. Kittrell and J. W. Eldridge, Ind. Eng. Chem. Res., 1990, 29, 321-324.

5 I. V. Kozhevnikov, Chem. Rev., 1998, 98, 171-198.

6 Y. Shiraishi, H. Hara, T. Hirai and I. Komasawa, Ind. Eng. Chem. Res., 1999, 38, 1589-1595.

7 P. Yang, S. Zhou, Y. Du, J. Li and J. Lei, RSC Adv., 2016, 6, 53860-53866.

8 L. Tang, G. Luo, M. Zhu, L. Kang and B. Dai, J. Ind. Eng. Chem., 2013, 19, 620-626.

9 P. M. Rao, A. Wolfson, S. Kababya, S. Vegac and M. V. Landau, J. Catal., 2005, 232, 210-225.

10 W. Jones, Catal. Today, 1988, 2, 357-367.

11 L. Chmielarz, P. Kuśtrowski, Z. Piwowarska, B. Dudek, B. Gil and M. Michalik, Appl. Catal., B, 2009, 88, 331-340.

12 L. Jin and B. Dai, Appl. Surf. Sci., 2012, 258, 3386-3392.

13 M. Motak, P. D. Costa and Ł. Kuterasiński, Catal. Today, 2011, 176, 154-158.

14 C. Marcos and I. Rodríguez, Appl. Clay Sci., 2014, 87, 219227.

15 S. S. G. Santos, H. R. M. Silva, A. G. D. Souza, A. P. M. Alves, E. C. D. S. Filho and M. G. Fonseca, Appl. Clay Sci., 2015, 104, 286-294.

16 C. Maqueda, A. S. Romero, E. Morillo and J. L. PérezRodríguez, J. Phys. Chem. Solids, 2007, 68, 1220-1224.

17 J. Temuujin, K. Okada and K. J. D. MacKenzie, Appl. Clay Sci., 2003, 22, 187-195.

18 J. Temuujin, K. Okada and K. J. D. MacKenzie, Appl. Clay Sci., 2003, 22, 187-195.

19 X. Zhang, G. Luo, M. Zhu, L. Kang, F. Yu and B. Dai, RSC $A d v .$, 2015, 5, 76182-76189.

20 B. Li, Z. Liu, C. Han, W. Ma and S. Zhao, J. Colloid Interface Sci., 2012, 377, 334-341.

21 R. Tian, O. Seitz, M. Li, W. Hu, Y. J. Chabal and J. Gao, Langmuir, 2010, 26, 4563-4566.

22 G. Luo, L. Kang, M. Zhu and B. Dai, Fuel Process. Technol., 2014, 118, 20-27.

23 D. Zhao, Y. Wang, E. Duan and J. Zhang, Fuel Process. Technol., 2010, 91, 1803-1806.

24 J. Qiu, G. Wang, D. Zeng, Y. Tang, M. Wang and Y. Li, Fuel Process. Technol., 2009, 90, 1538-1542. 